\title{
Les dangers de la décentralisation gouvernementale : la déresponsabilisation face aux inégalités régionales
}

\author{
Bruno Jean \\ Université du Québec à Rimouski
}

$R$ éussir la décentralisation gouvernementale au Québec ${ }^{1}$ nécessite de comprendre et de maîtriser les dangers associés à la décentralisation. Un des dangers majeurs, c'est la déresponsabilisation des élites sociopolitiques et des citoyens des régions face au destin de la collectivité globale, et leur déresponsabilisation face à la croissance des inégalités ou des disparités régionales amplifiées par la décentralisation elle-même. Toutes les régions ne sont pas sur la même ligne de départ de la décentralisation à la sauce néo-libérale qui se profile à l'horizon. Tous les territoires du Québec n’ont pas un stock équivalent de ressources naturelles sur lesquelles toucher des redevances ou encore des niveaux comparables d'activité économique sur lesquels lever des impôts. Au Québec, le débat sur la décentralisation est paradoxal; elle est réclamée par des élites locales et régionales qui apparaissent par ailleurs bien frileuses quand vient le temps d'exercer de nouvelles responsabilités.

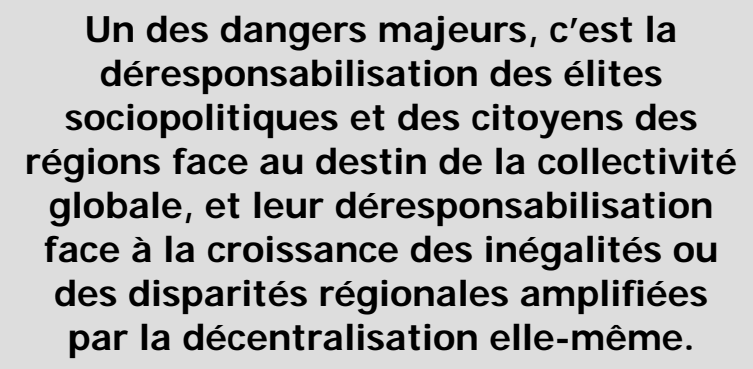

Une autre demande de décentralisation vient des attentes citoyennes pour une meilleure participation à la prise de décision sur les affaires publiques au-delà de la simple démocratie représentative. Mais les dangers de la décentralisation sont aussi du côté de l'État qui pourrait abandonner ses propres responsabilités vis-à-vis du développement régional dont on peut se demander s'il ne serait pas mieux servi par un gouvernement central qui en aurait fait sa véritable priorité politique. Notre propos se termine en examinant quelques conditions de la décentralisation dans la perspective d'un développement territorial solidaire, soit un développement qui tient compte du poids démographique urbain et de la nouvelle solidarité rurale-urbaine qui doit en être un des principes. La mise en place d'une sorte de gouvernement régional «intermédiaire » élu apparaît également une condition de réussite de la décentralisation.

\section{Le paradoxe de la décentralisation}

D’entrée de jeu, il faut mettre au jour ce paradoxe de la décentralisation. Elle semble souhaitée afin que les décideurs régionaux aient davantage de capacité d'action pour réduire les disparités régionales dont ils se sentent les victimes, par exemple en agissant sur l'occupation et l'aménagement du territoire et sur l'attractivité socio-économique de leur région. Mais par ailleurs, la décentralisation crée aussi les conditions pour générer de nouvelles disparités qui viendraient, en quelque sorte, annuler ses effets positifs. Cela dit, nous persistons à penser que le développement des territoires du Québec suppose une approche territoriale du développement $^{2}$ impliquant la mobilisation des acteurs régionaux et leur implication effective dans la prise de décision sur les affaires les concernant, évidemment dans un modèle de gouvernance régionale à construire, mais reposant sur un partenariat inédit entre le secteur privé, le secteur public et ce qu'on appelle maintenant la société civile.

Il faut sans doute répondre à cette attente citoyenne, insatisfaite par la démocratie représentative, de participation plus effective aux décisions de l’État concernant des domaines aussi variés que la gestion des forêts publiques, la protection de l'environnement ou l'adaptation du système scolaire à la nouvelle économie du savoir. Personnellement, je suis enclin à pen- 
ser qu'il y a une profonde confusion entre les attentes d'une démocratie participative ou citoyenne, cristallisées dans les propos du Forum social mondial, et celles qu'on attribue à une demande de décentralisation (et exprimées si largement dans les mémoires déposés à la Commission Bélanger-Campeau ou lors des tournées de Solidarité rurale). Pour plusieurs observateurs, la participation citoyenne, et la nouvelle gouvernance démocratique, passe par la décentralisation, mais cela n’est guère démontré à mon sens.

Si la déresponsabilisation des acteurs régionaux est un danger réel propre à la décentralisation - et à entendre le discours de certains élus locaux maintenant réunis dans des Conseils régionaux d'élus, ce danger est bien réel -, il en existe un autre tout aussi menaçant. C'est le danger de la déresponsabilisation de l'État qui est tenté de mettre en œuvre une forme de décentralisation où il se délestera de certains tâches ou responsabilités en faveur des régions ou des MRC sans transférer les ressources fiscales pour assumer ces nouvelles compétences. D’ailleurs, on peut s'inquiéter que la décentralisation réelle en soit surtout une qui se fasse davantage par délestage des fonctions étatiques gérées centralement que par construction d'une véritable capacité de gestion administrative et politique des territoires visés par cette décentralisation.

Ceci nous conduit évidemment à introduire un autre danger de la décentralisation; elle suppose que les acteurs (région, MRC ou municipalité locale) maîtrisent non seulement une capacité financière, mais un aussi une capacité gestionnaire. On peut craindre que de telles capacités soient trop peu développées dans plusieurs régions. La remise aux municipalités locales de la voirie tertiaire est probablement passée inaperçue aux yeux du grand public, d'autant que cela semblait faire sens. Mais entretenir quelques kilomètres de route s'est avéré une aventure fort coûteuse pour certaines localités; de plus, la voirie, même tertiaire, demande parfois une expertise en génie civil qui est hors de portée d'une petite localité.

\section{La décentralisation et le défi d'un développement territorial solidaire}

Depuis des décennies, on entend un discours «politiquement correct » sur le développement régional au Québec; on voudrait que les élites régionales soient les mieux placés pour assurer le développement des régions. Dans ce contexte, de nombreuses voix se font entendre pour vanter les mérites de la décentralisation $^{3}$. Les diverses expériences menées à travers le monde ont pourtant montré qu'une décentralisation bien comprise s'accompagne non seulement de nouvelles compétences ou responsabilités, mais aussi d'enveloppes budgétaires appropriées et ce, dans un souci de réduction des disparités.

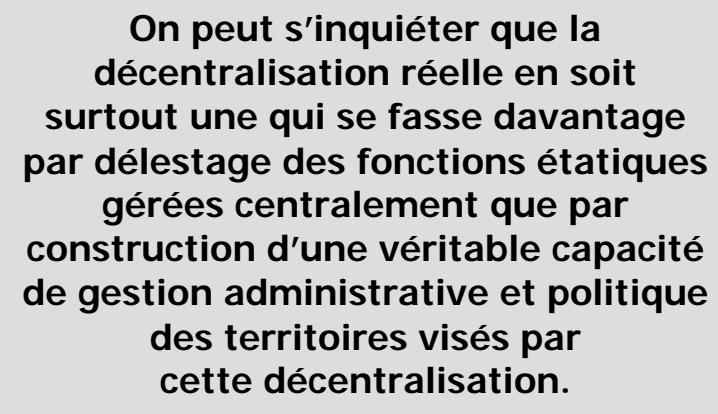

L'autre type de décentralisation, encore plus dangereuse, consiste à transférer des responsabilités en demandant aux régions de subvenir elles-mêmes, comme avec les redevances sur l'exploitation forestière, à leurs propres besoins. Une telle approche risque d'exacerber les disparités régionales alors qu'on veut justement les atténuer. Et il en est de même pour ce nouveau discours politique qui désire inscrire ces inégalités dans la dynamique de la concurrence néolibérale où chaque région devrait identifier et tabler sur sa spécialisation distinctive.

$\mathrm{Au}$ risque de paraître complètement irrévérencieux pour ces belles idées sur la régionalisation, il faut se demander si le «développement régional » ne serait pas mieux servi par un gouvernement central qui en aurait fait réellement sa priorité. À cet égard, le modèle québécois de développement, issu de la Révolution tranquille, avec la modernisation de l'appareil d’État et sa décentralisation, voire sa régionalisation comme dans le cas de l'éducation et de la santé, avec la création des sociétés d'État actives sur le plan économique, avec sa philosophie d'équité sociale, etc., a certainement permis une réduction sensible des écarts de développement entre les régions du Québec. Certains ne manqueront de rappeler les limites de ce modèle pour assurer le plein développement des territoires du Québec, avec l'approche sectorielle qui caractérise l'action publique gouvernementale et l'approche territoriale que réclamerait le développement régional bien compris. 
Il n’en reste pas moins que, pour une région donnée, il peut être fort avantageux que certains arbitrages sur les choix publics soient faits au niveau central ou national, ce qui évite des discussions et des conflits internes, épuisants et mettant à mal une cohésion sociale régionale déjà fragile. Si la décision prise centralement ne leur est pas favorable, les acteurs régionaux, au lieu de s'entre-déchirer, pourront au moins identifier un adversaire commun, principe d'opposition fort utile pour renforcer une identité locale ou régionale. Mais le principal problème, c'est qu'aucun gouvernement central n'a réellement fait le choix des régions, ou pris le parti des régions. D’ailleurs, cela revient peut-être à mettre en œuvre une «subsidiarité » bien comprise.

La généralisation de la sensibilité environnementale offre une opportunité unique pour renouer un nécessaire dialogue rural-ubain, ou le dialogue entre la métropole et les régions. Il est temps de penser à un nouveau contrat social entre les producteurs (en région) et les consommateurs (en métropole). Les uns et les autres, au lieu de se poser en adversaires, pourraient alors devenir les partenaires d'une nouvelle dynamique de développement solidaire ${ }^{4}$, selon un modèle québécois de développement territorial se construisant sur la base d'une meilleure compréhension des rapports urbains-ruraux, à l'heure de la nouvelle économie et du développement durable. Mais je suis conscient ici qu'en posant ces rapports en ces termes, on tombe dans un piège, celui d'enfermer plusieurs régions dans un rôle de production de biens pour les autres, sans la capacité reconnue de faire épanouir dans de ces espaces de véritables sociétés locales ou régionales 5 .

\section{II faut envisager la création d'une véritable structure gouvernementale intermédiaire élue.}

Le développement des vastes régions du Québec, souvent rurales, ne pourra se faire qu'avec les seules ressources de ces territoires généralement fort peu peuplés. Le développement de tous les territoires du Québec devient alors une question qui interpelle la société québécoise dans son ensemble et non seulement les citoyens des régions en question. Il faut passer d'un certain égoïsme territorial à un développement territorial solidaire qui rend caduque la discus- sion sur des "redevances", une approche qui va plutôt engendrer un nouveau Québec cassé en deux : celui des villes dynamiques et celui d'une ruralité survivant chichement de redevances sur des ressources qui créent de la prospérité ailleurs.

Un large travail d'éducation sociopolitique reste à faire pour montrer les avantages réciproques d'une approche partenariale en faveur de ce qui deviendrait un grand projet de société au Québec, soit le développement territorial solidaire. Cela veut dire qu'une partie des ressources fiscales des villes vont servir aux gouvernements pour maintenir et développer des services dans des zones rurales à faible densité, mais, en retour, ces zones pourront continuer à offrir aux populations urbaines de nombreuses aménités rurales appréciées par les citadins.

\section{Une condition préalable : la création d'une structure intermédiaire élue}

Si l'on souhaite s'engager dans une véritable décentralisation, et non dans une régionalisation ou encore une déconcentration administrative, comme on le constate partout dans le monde où l'on a assisté à une décentralisation, il faut envisager la création d'une véritable structure gouvernementale intermédiaire élue. Des élus possédant la légitimité pour gérer la décentralisation, pour prendre des décisions concernant des dépenses publiques financées des ressources monétaires contrôlées régionalement.

Une telle structure intermédiaire n'existe pas au Québec, les MRC sont trop petites, mais certaines villes de plus de 100000 habitants possèdent ou peuvent se donner rapidement des capacités financières et gestionnaires pour accueillir une véritable décentralisation. Certaines de ces villes, et notamment les plus grandes, sont prêtes et souhaitent même un tel virage qui pourraient bien se faire avec une dynamique qui implique le gouvernement fédéral. Cependant, comme on le constate en maints endroits, les rapports urbainsruraux ne sont pas toujours des plus harmonieux là où des villes-centres dominent les instances comme les MRC. Et d'ailleurs, une telle perspective qu'on voit se dessiner avec les réformes municipales récentes laisse fort inquiètes les autorités de nos 53 MRC rurales (c'est-à-dire sans AR pour signifier une agglomération de recensement, soit une ville de 30000 habitants et plus) et qui représentent sans doute près des troisquarts du territoire québécois. 
Par ailleurs, il faut aussi se demander quelles compétences ou responsabilités devraient alors être décentralisées au niveau de cette instance intermédiaire élue. Certains prétendent que le gouvernement du Québec n'a pas grand chose à décentraliser et que le véritable danger, c'est de réaliser une décentralisation avant d'avoir réglé la question constitutionnelle. Ce n'est pas pour rien qu'on parle d'une décentralisation à la carte, sur mesure, la variance régionale étant relativement forte encore de nos jours. On peut alors imaginer que l'agenda politique de telle ou telle région pourrait différer sensiblement. Et il resterait un problème de taille : comment concilier la responsabilité avec la solidarité ? Car, faut-il le rappeler, le principal danger avec la décentralisation, c'est de mettre en œuvre une mauvaise décentralisation, et la mauvaise décentralisation, c'est celle qui ne serait pas négociée avec les partenaires territoriaux, c'est celle qui serait dictée unilatéralement par un État se soustrayant lui-même à ses responsabilités fondamentales. Au nombre de celles-ci, depuis des décennies, il y a la réduction des disparités régionales de développement; la lutte à ces disparités peut paradoxalement passer par la valorisation des atouts des diverses régions, et donc par des approches de développement qui cultivent ces différences régionales.

\section{Notes et références}

1 Version remaniée d'une conférence lors du Forum des territoires du Québec sur les dangers de la décentralisation gouvernementale Québec, Mouvement Territoires et Développement (MTD), 8 septembre 2004.

2 Une telle approche est développée par le Centre de recherche sur le développement territorial dont on peut connaître les travaux en visitant son site Internet au www.uqar.qc.ca/ crdt.

3 Jean, B. (2003), « Réussir le développement approprié du territoire gaspésien : quelques conditions nécessaires mais sans doute non suffisantes », Gaspésie, vol. 40, n² 2, Gaspé, Musée de la Gaspésie, p. 32-36.

4 Ces propos ont été exprimés dans un article paru dans $L e$ Devoir; voir : Jean, B. (2003), « Le développement territorial : de l'égoïsme à la solidarité », 20 mai, p. A7.

5 Voir Polèse, M., R. Shearmur et al. (2002), La périphérie face à l'économie du savoir : la dynamique spatiale de l'économie canadienne et l'avenir des régions non métropolitaines du Québec et des provinces de l'Atlantique, Montréal, INRS-Urbanisation, Culture et Sociétés, 327 p. 